\title{
Agronegocios
}

\section{SARS-CoV-2 y cambios en la vida de los estudiantes de Economía Agrícola y Agronegocios \\ SARS-CoV-2 and changes in agricultural}

Economics and Agribusiness Student's Life

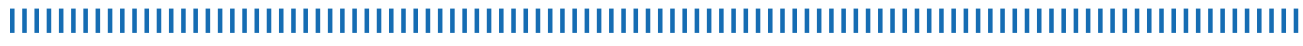

Amanda María Naranjo Retana’ Alejandra María Rodríguez Rodríguez ${ }^{2}$

Eilyn González Sánchez ${ }^{3}$ Fauricio Redondo Angulo ${ }^{4}$ Stephanie Sánchez León ${ }^{5}$ Ericka Cortés Arias ${ }^{6}$ Abril Rivas Briceño7

Fecha de recepción: 01 de mayo, 2021 Fecha de aprobación: 13 de julio, 2021

Vol.7 N² Julio- diciembre 2021

Naranjo, A. et al. (2021). SARS-CoV-2 y cambios en la vida de los estudiantes de Economía Agricola y Agronegocios. Revista e-Agronegocios, 7(2). https://revistas.tec.ac.cr/index.php/eagronegocios/

article/view/5690 


\section{Agronegocios}

${ }^{1}$ Universidad de Costa Rica, San José, Costa Rica. Correo electrónico: amanda.naranjo@ucr.ac.cr

(iD https://orcid.org/0000-0002-3105-2968

2Universidad de Costa Rica, San José, Costa Rica. Correo electrónico:

alejandra.rodriguezrodriguez@ucr.ac.cr

(iD https://orcid.org/0000-0002-5460-0576

3 Universidad de Costa Rica, San José, Costa Rica.

Correo electrónico: eilyn.gonzalezsanchez@ucr.ac.cr

(iD) https://orcid.org/0000-0002-1775-957X

${ }^{4}$ Universidad de Costa Rica, San José, Costa Rica. Correo electrónico: fauricio.redondo@ucr.ac.cr

(iD https://orcid.org/0000-0001-9241-5462

5 Universidad de Costa Rica, San José, Costa Rica. Correo electrónico: stephanie.sanchezleon@ucr.ac.cr

(iD https://orcid.org/0000-0002-5768-3768

- Universidad de Costa Rica, San José, Costa Rica. Correo electrónico: ericka.cortes@ucr.ac.cr

(iD https://orcid.org/0000-0002-7738-3551

7 Universidad de Costa Rica, San José, Costa Rica. Correo electrónico: abril.rivas@ucr.ac.cr

(iD https://orcid.org/0000-0002-5609-6238 


\section{Resumen}

El objetivo de este artículo es identificar y analizar los cambios en la vida cotidiana, laboral y académica que han percibido los y las estudiantes de Economía Agrícola y Agronegocios de la Universidad de Costa Rica (UCR) pertenecientes a la sede Rodrigo Facio, sede del Sur y el recinto de Guápiles, producto de la adopción de la modalidad virtual en su proceso de aprendizaje. Se seleccionó una muestra de estudiantes activos y se les aplicó una encuesta online a través de la herramienta de Google Forms. A raíz de la virtualidad los estudiantes percibieron afectación en su salud física y mental, así como se evidenció que persisten las brechas que limitan la inclusividad de los estudiantes para llevar a cabo sus estudios de manera virtual.

Palabras clave: COVID-19, salud mental, desigualdad digital, adaptación, estadística.

\section{Abstract}

The objective of this article is to identify and analyze the changes in daily, labor, and academic life that have been perceived by the students of Agricultural Economics and Agribusiness of the University of Costa Rica (UCR) belonging to the Rodrigo Facio campus, South campus, and the enclosure of Guápiles, through the implementation of the virtual modality, on the methodological design. A sample of active students was selected, and an online survey was applied through the Google Forms tool. As a result of virtuality, the students have perceived affectation in aspects such as the mental and physical health. It was also evidenced that there are gaps that limit the inclusiveness of students to carry out their studies virtually.

Key words: COVID-19, mental health, digital inequity, adaptation, statistics. 


\section{Introducción}

El COVID-19 presentó una velocidad de expansión y gravedad considerable, por lo que el 11 de marzo del 2020, la Organización Mundial de la Salud (OMS) lo enunció como pandemia (Johnson, Saletti, Tumas, 2020). En Costa Rica el primer caso de COVID-19 se confirmó el 6 de marzo y diez días después se emitió la emergencia nacional, la cual trajo cambios en la vida de los costarricenses, pues se estableció una serie de medidas sanitarias (Gaiser, 2020). Uno de los sectores que más ha sufrido cambios en el país es el de la educación, ya que las medidas de confinamiento, para minimizar la propagación del virus, obligaron a cambiar el ambiente presencial por la virtualidad, lo cual ha afectado el ritmo de trabajo y educación.

En Costa Rica la enseñanza de estudios superiores se ha impartido mayoritariamente de manera presencial. Sin embargo, con el rápido desarrollo de la tecnología poco a poco se han venido incorporando nuevas herramientas tecnológicas. La educación a distancia utilizando entornos virtuales es de vital importancia para el sistema educativo, ya que se encuentra en un momento donde hay grandes cambios y se "requiere de la construcción de nuevos roles, nuevos espacios y nuevas competencias comunicativas, pedagógicas y tecnológicas" (Peña et al., 2012, p.121). Pero más allá de querer ahondar en el tema de la enseñanza virtual, el objetivo primordial es saber cuáles son los cambios que esta puede provocar en la vida cotidiana, laboral y académica de los estudiantes. Por tanto, es considerable conocer la perspectiva de los estudiantes de Economía Agrícola de la UCR en relación con la modalidad virtual que ha implementado esta casa de enseñanza para salir a flote con la educación superior en el país. Esta investigación permitirá analizar los cambios que han ocasionado los semestres virtuales en la vida cotidiana, laboral y académica de los estudiantes activos de la carrera de Economía Agrícola y Agronegocios de dicha institución.

\section{Referente Teórico}

El COVID-19 ha afectado de manera indirecta en estudiantes universitarios, esto se debe a la migración a la educación virtual (Montalvo \& Montiel, 2020). Se han realizado estudios que muestran los cambios cotidianos de los estudiantes especialmente en el ámbito social y de salud. En el primer caso en el estudio de la UNESCO, se muestra cómo los estudiantes han tenido que reorganizar su vida cotidiana para adaptarse a la virtualidad, también se expresa que "la pérdida de contacto social y de las rutinas de socialización que forman parte de la experiencia cotidiana de un estudiante de educación superior tendrán un costo" (UNESCO, 2020, p.16).

La pandemia también ha generado implicaciones en la salud mental, en un estudio se pudo comprobar que los niveles de ansiedad de los estudiantes universitarios de China subieron un 24,9\% a raíz de la pandemia, y algunos de los factores que afectaron 
fueron: no vivir con los padres, vivir en zonas rurales, no tener un ingreso económico estable, el contagio por COVID-19 en el núcleo familiar y la ausencia de la comunicación interpersonal (Cao et al., 2020). Asimismo, existen estudios en donde se muestra que la población joven presentó mayores niveles de estrés, ansiedad y depresión debido a la pandemia y como las instituciones educativas tienen un rol fundamental para la implementación de programas de intervención para disminuir dichos niveles (Ozamiz et al., 2020).

También hay evidencia de cambios en la salud física, en un estudio a nivel mundial, se hace referencia a que el aislamiento social ha tenido una repercusión en los niveles de actividad física, se realizó una comparación con los 30 millones de usuarios de los monitores de movimiento Fitbit a nivel mundial en la actividad física, de marzo de 2019 y marzo 2020, se observó una disminución entre 7\% a 38\% en la cantidad de pasos caminados diariamente (Celis et al., 2020). Asimismo, en el caso de los estudiantes, existen estudios, en donde se realizó una encuesta a una muestra de 267 estudiantes de la Universidad Católica de Cuenca Sede Azogues ubicada en Ecuador, en este estudio un 25,1\% de los estudiantes manifestó no realizar actividad física durante la cuarentena, sin embargo, del porcentaje restante un 21,7\% expresó realizar actividad física principalmente para estar en forma (Buri et al., 2020).

Las investigaciones que analizan los cambios académicos producto de la pandemia detallan aspectos como la conexión, la modalidad implementada y el rendimiento. En otro estudio se busca describir el efecto de la educación virtual, esto mediante la aplicación de encuestas a una muestra de una población de 260 estudiantes de Universidades de Perú, tanto públicas como privadas (Huanca et al., 2020). En este estudio se evidenciaron las brechas causadas por la falta de habilidades o herramientas tecnológicas por parte de estudiantes y docentes. Por su parte en un estudio de la CEPAL se evidenció que muchas instituciones educativas en América Latina y el Caribe no cuentan con la infraestructura necesaria para la implementación de tecnologías y además se muestra una diferencia de acceso a estas entre zonas urbanas y rurales (CEPAL, 2020). La UNESCO indica que el porcentaje de hogares con conexión a internet para América Latina es de un 45\%, lo cual evidencia una problemática para aquellos estudiantes que no cuentan con conexión (UNESCO, 2020).

Por otro lado, el rendimiento de 458 estudiantes de la Universidad Autónoma de Madrid (España) ha mejorado por distintas razones, principalmente por la nueva metodología utilizada, la cual posibilita que los estudiantes tengan a disposición la materia en cualquier momento (Gonzalez et al., 2020). Asimismo, la modalidad virtual tiene ventajas como las grabaciones de las clases, poder retroceder los videos en incluso descargarlos, no obstante, evidencia que hay desventajas que se deben considerar como la dependencia a internet y la falta de concentración o compromiso que puede generar esta modalidad por parte de los estudiantes (De Oliveira-Dias, De Oliveira-Albergarias-Lopes, Correia-Teles, 2020). 
Además, para un estudio se realizó un cuestionario a 548 estudiantes de la Universidad de Extremadura, en donde para un 75\% de los estudiantes la virtualidad ha exigido una mayor dedicación en comparación con la modalidad presencial y un 51,6\% de estos manifestó no estar aprendiendo con las clases virtuales (Pérez-López, Atochero, Rivero, 2021). De ahí que uno de los principales retos que implica la virtualidad es la autodisciplina (Aristovnik et al., 2020). En Costa Rica también se ha analizado el efecto de la virtualidad en los estudiantes de la UCR ante el COVID-19, por ejemplo, un estudio describe la perspectiva estudiantil de diferentes escuelas, facultades y sedes de la UCR ante la virtualización de los cursos, en donde se permite tener una referencia acerca de cuál modalidad se utilizó más o cuál despertó mayor interés en los estudiantes, o la disposición de mantener ciertos cursos de manera virtual, por ejemplo , 6 de cada 10 estudiantes indicaron que el curso podría seguirse impartiendo de manera virtual (Kikut, 2020).

\section{Metodología}

La población objetivo del presente estudio fueron los 596 estudiantes de bachillerato activos durante el primer semestre del 2020 de la carrera de Economía Agrícola y Agronegocios de la sede Rodrigo Facio y la sede del Sur, además del recinto de Guápiles de la Universidad de Costa Rica, donde también se imparte la carrera. Se estimó un tamaño de muestra empleando la fórmula de muestreo irrestricto, correspondiente a proporciones para poblaciones finitas, que según Mora (2015) se define como en la (Ecuación 1):

$$
n=\frac{N\left(z_{\alpha / 2}\right)^{2} P Q}{\left(e^{2}(N-1)\right)+\left(\left(z_{\alpha / 2}\right)^{2} P Q\right)}
$$

Donde:

- $n$ es el tamaño de la muestra,

- $\quad N$ es el tamaño de la población,

- $Z_{\alpha / 2}$ valor de la distribución normal estándar asociado al nivel de significancia,

- P es la proporción esperada,

- $Q$ es $1-P ; \mathrm{y}$

- $e$ corresponde al error de muestreo máximo permitido.

En la estimación del tamaño de muestra se utilizó una significancia del 5\%, un error de muestreo del 10\% y con $\mathrm{P}=\mathrm{Q}=0,5$. Con lo anterior, el tamaño de muestra resultó de 83 personas, detallándose en el Cuadro 1. 
Cuadro 1. Parámetros para la estimación del tamaño de la muestra.

\begin{tabular}{lcc}
\hline Parámetro & Nomenclatura & Valor \\
\hline Significancia & $\alpha$ & 0,05 \\
Desviaciones típicas & $z_{\alpha / 2}$ & 1,96 \\
Error & $e$ & 0,10 \\
Población total & $N$ & 596 \\
Proporción esperada 1 (P) & $P$ & 0,50 \\
Proporción esperada 2 (Q) & $Q$ & 0,50 \\
Tamaño de la muestra (población finita) & $N$ & 82,83 \\
\hline
\end{tabular}

Fuente: elaboración propia.

Se diseñó una encuesta y se aplicó a través del instrumento de Google Forms, esta contenía una introducción al tema, posteriormente, las preguntas se dividieron en cuatro secciones, información general, preguntas laborales, vida cotidiana del estudiante y preguntas de carácter académico. Dicha encuesta fue habilitada el día 6 de noviembre de 2020 y se cerró el día 13 de noviembre del mismo año, se obtuvieron 98 respuestas. En el cuadro 2 se observa la distribución de la población y la muestra por sede o recinto.

Cuadro 2. Distribución de la población y la muestra según sede o recinto.

\begin{tabular}{lcccc}
\hline \multicolumn{1}{c}{ Sede o Recinto } & Población & Porcentaje & Muestra & Porcentaje \\
\hline Recinto de Guápiles & 129 & 22 & 29 & 30 \\
Sede Rodrigo Facio & 380 & 64 & 63 & 64 \\
Sede del Sur & 87 & 15 & 6 & 6 \\
Total & $\mathbf{5 9 6}$ & $\mathbf{1 0 0}$ & $\mathbf{9 8}$ & $\mathbf{1 0 0}$ \\
\hline
\end{tabular}

Fuente: elaboración propia.

Para el análisis estadístico de los datos se realizó un ANOVA, sin embargo, al no cumplirse los supuestos que la sustentan, se llevó a cabo una prueba no paramétrica. Se efectuaron comparaciones entre las poblaciones de estudiantes según sede o recinto mediante la prueba de Kruskal Wallis (1952), se creó una variable dicotómica que registra si el o la estudiante cambió de residencia durante la pandemia y mediante la prueba de Wilcoxon (1945) se efectuaron comparaciones según esta condición. La heterocedasticidad se verificó mediante la prueba de Levene (1960) y todo se trabajó al $5 \%$ de significancia. Para el análisis se utilizaron los programas Microsoft Office Excel versión 2016, R versión 3.6.1 y GeoDa versión 1.16. 


\section{Resultados}

\section{Aspectos generales}

De acuerdo con el análisis realizado a los datos recolectados en la primera parte de la encuesta aplicada a los estudiantes, se obtuvieron los siguientes resultados. En cuanto a los aspectos generales se realizó un recuento de los estudiantes según año de ingreso (Figura 1) y un registro de sus residencias durante el periodo estudiado (Figura 2, 3 y 4).

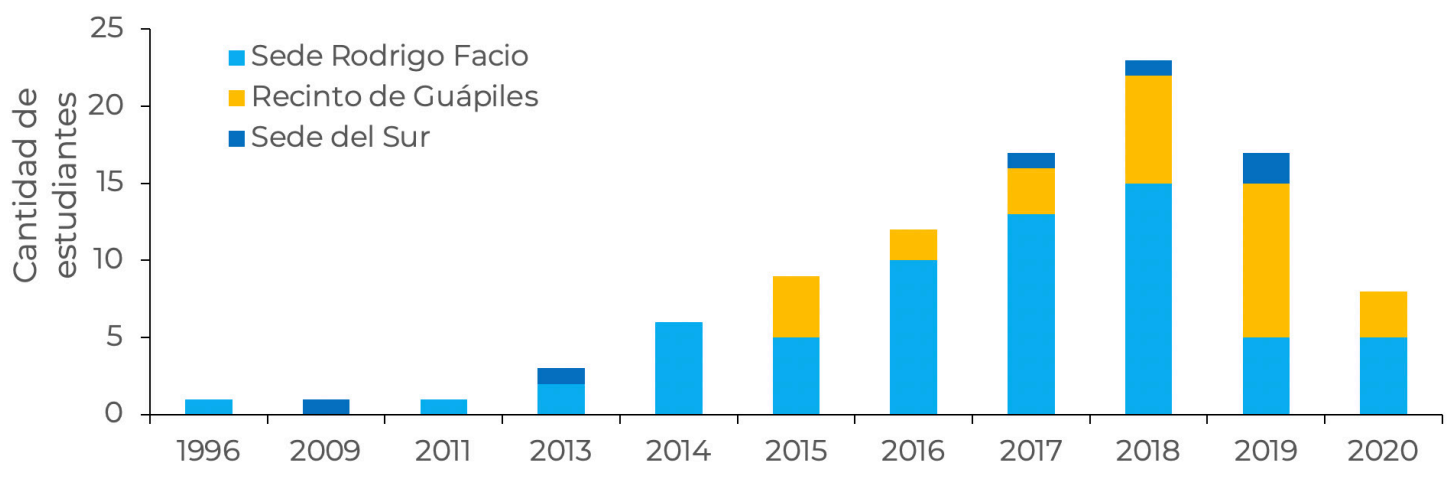

Figura 1. Cantidad de estudiantes encuestados según año de ingreso a la Universidad por sede o recinto

Fuente: elaboración propia.
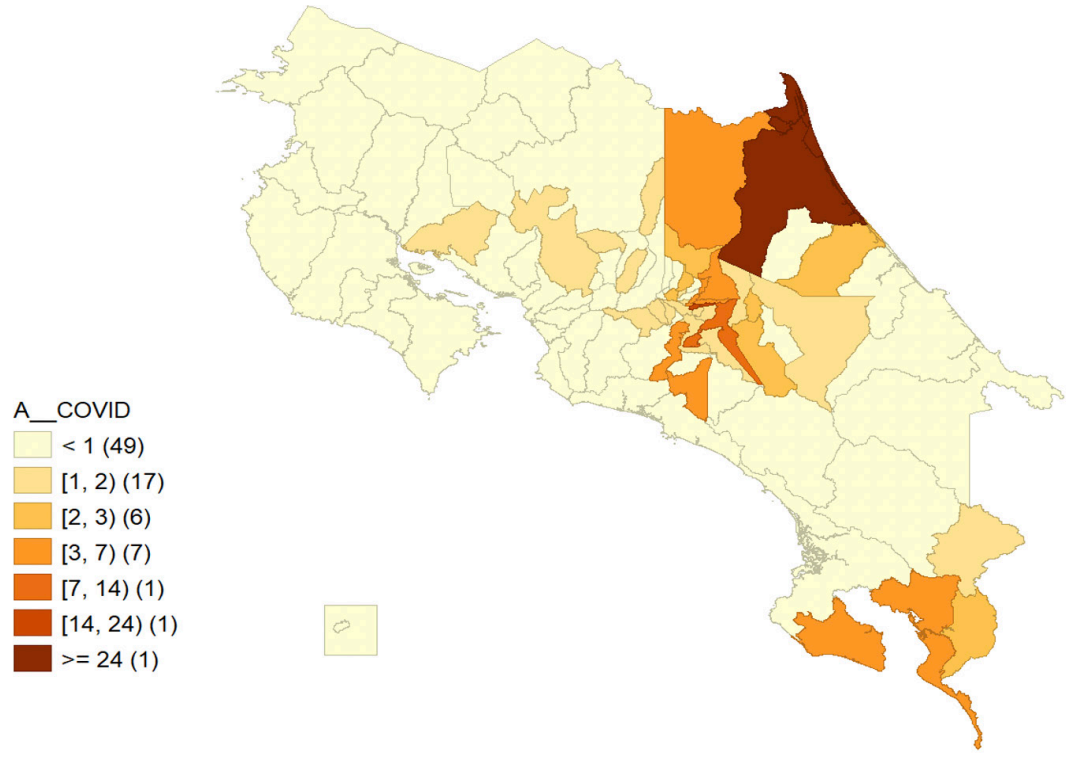

Figura 2. Distribución de los estudiantes en el territorio costarricense antes de la pandemia.

Fuente: elaboración propia. 

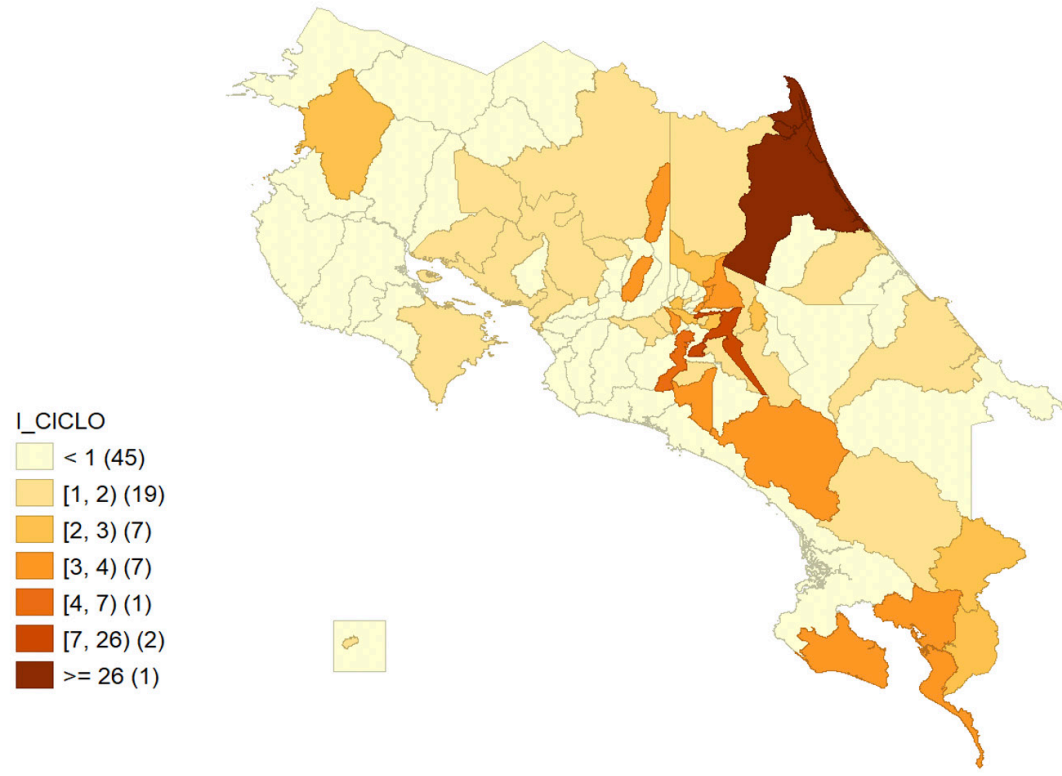

Figura 3. Distribución de los estudiantes en el territorio costarricense durante el semestre I 2020.

Fuente: elaboración propia.
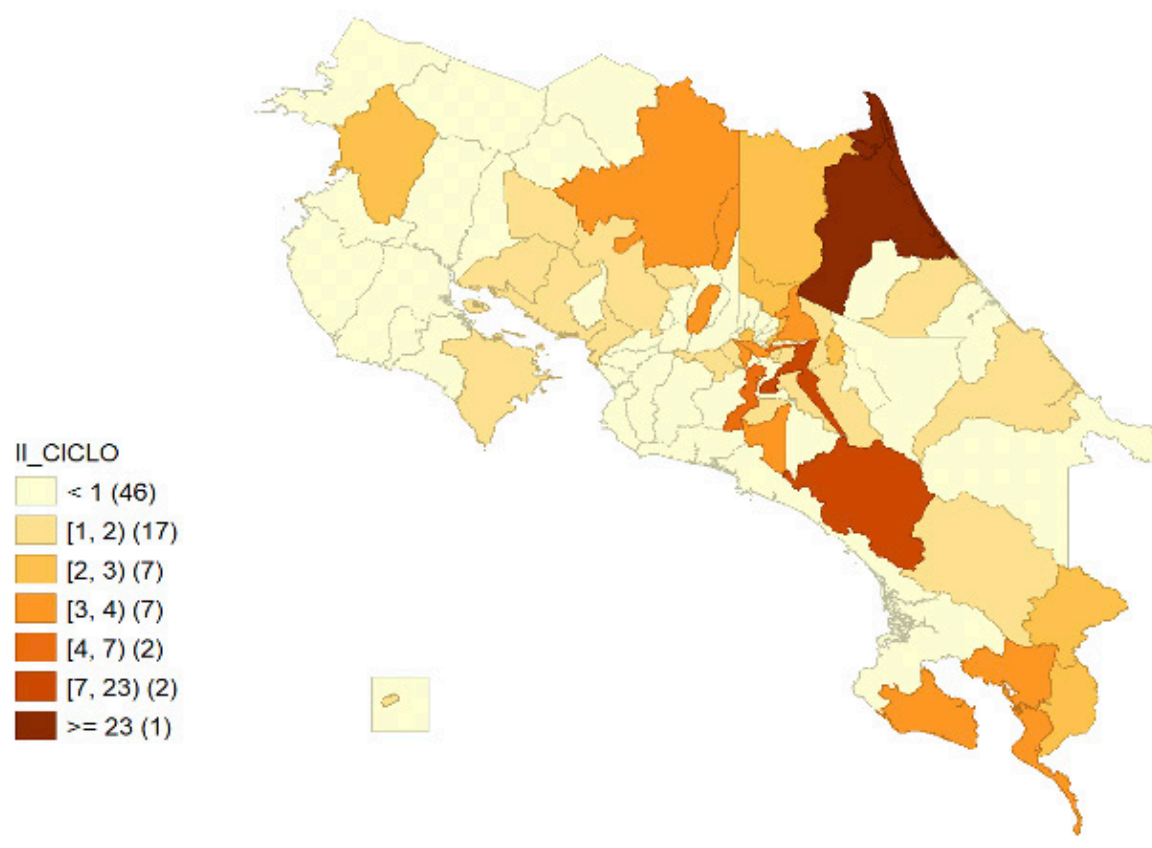

Figura 4. Distribución de los estudiantes en el territorio costarricense durante el semestre II 2020.

Fuente: elaboración propia. 


\section{Aspectos laborales}

Referente a los aspectos laborales se buscó identificar la situación laboral de los estudiantes antes de la pandemia, durante el I y II semestre de 2020, esto con el propósito de conocer si habían experimentado cambios en su condición laboral (Figura 5).

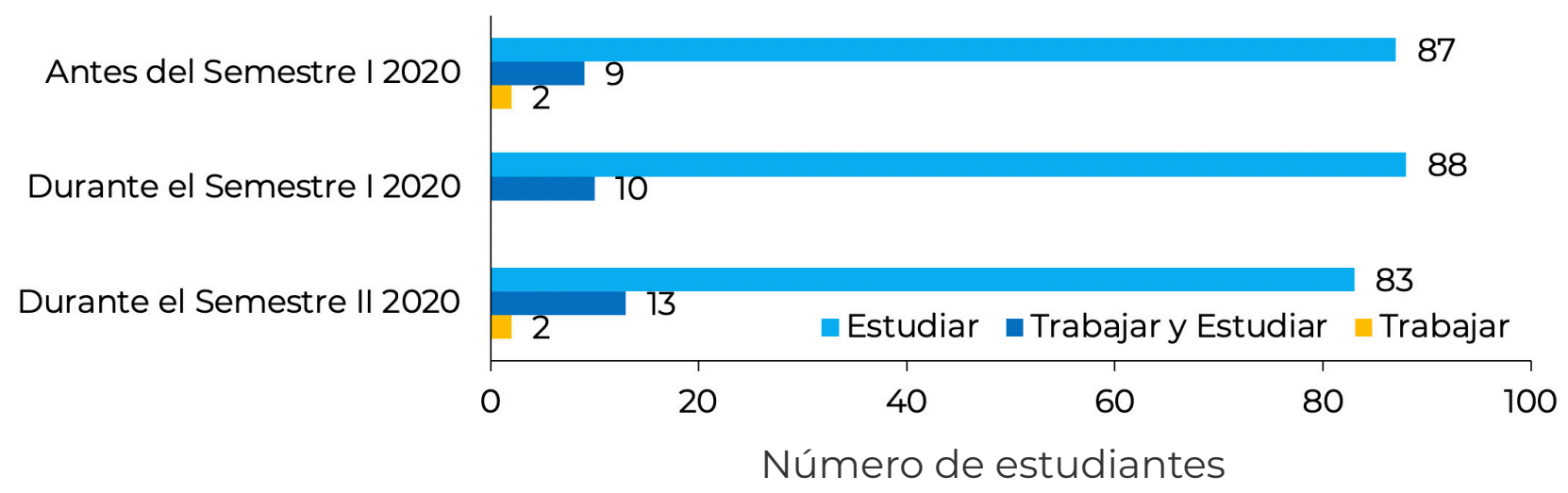

Figura 5. Situación laboral de los estudiantes de Economía Agrícola y Agronegocios antes del Semestre I 2020, durante el Semestre I 2020 y después del Semestre I 2020. Fuente: elaboración propia.

De acuerdo con los resultados obtenidos de las preguntas orientadas a estudiar y trabajar, los estudiantes expresaron que pueden disponer de más tiempo al no tener que trasladarse a la Universidad de Costa Rica, y que debido a las clases asincrónicas pueden organizar su tiempo a conveniencia e incluso algunos han empezado a trabajar en negocios familiares.

\section{Aspectos cotidianos}

Para todos los aspectos evaluados, al comparar entre sedes y recintos, así como al comparar según la condición de residencia, no se encontraron diferencias significativas $(p>0,05)$ (Cuadro 3). Además, se resalta que un $51 \%$ de los encuestados manifestaron tener la oportunidad de invertir tiempo en otras actividades debido a la pandemia, dentro de las más mencionadas fue realizar actividad física como ejercicio o algún deporte, o actividades recreativas como leer, cocinar, pintar, e incluso 3 personas mencionaron la oportunidad de crear huertas. 
Cuadro 3. Resumen de estadística de la variable de afectación de los estudiantes según actividad.

\begin{tabular}{|c|c|c|c|c|c|c|c|c|c|}
\hline \multirow{2}{*}{ Actividad } & \multirow{2}{*}{ Promedio } & \multirow{2}{*}{ Moda } & \multirow{2}{*}{ Min } & \multirow{2}{*}{ Max } & \multirow{2}{*}{$\mathrm{DE}$} & \multirow{2}{*}{ CV\% } & \multirow{2}{*}{$\mathrm{n}$} & \multicolumn{2}{|c|}{ Probabilidad } \\
\hline & & & & & & & & Casol & Caso2 \\
\hline Pasatiempos & 6,38 & 10 & 1 & 10 & 2,91 & 45,58 & 92 & 0,60 & 0,14 \\
\hline Relaciones Interpersonales & 6,25 & 8 & 1 & 10 & 2,92 & 46,74 & 94 & 0,98 & 0,58 \\
\hline Horarios de sueño & 6,10 & 10 & 1 & 10 & 3,28 & 53,82 & 92 & 0,61 & 0,35 \\
\hline Horarios de comida & 5,30 & 1 & 1 & 10 & 3,42 & 64,52 & 93 & 0,49 & 0,14 \\
\hline Tiempo en familia & 5,13 & 1 & 1 & 10 & 3,17 & 61,81 & 92 & 0,70 & 0,86 \\
\hline
\end{tabular}

Nota. Caso 1 = Probabilidad según la prueba de Kruskal Wallis para la comparación de los estudiantes entre sedes y recinto, Caso 2 = Probabilidad según la prueba de Wilcoxon para la comparación entre estudiantes que cambiaron de residencia y los que no cambiaron.

Fuente: elaboración propia.

Con respecto al tema de la salud mental y física, un 73\% de los estudiantes expresaron que las clases virtuales les han provocado ansiedad, estrés, cambios de humor, depresión, problemas de concentración e insomnio. Simultáneamente, un 86\% de los estudiantes manifestaron una afectación en su salud física principalmente por el sedentarismo que presentan los estudiantes al permanecer frente a una computadora por largos periodos de tiempo, siendo el dolor de cabeza, cuello, espalda, rodillas y problemas de visión, los principales malestares manifestados.

\section{Aspectos académicos}

En cuanto al aspecto académico, según los datos recopilados, cerca del 90\% de los encuestados matriculó entre cuatro y ocho cursos en el primer ciclo del 2020, dejando apenas un $10 \%$ de encuestados con tres o menos cursos matriculados. Además, un $21 \%$ de los estudiantes manifestó retirar al menos un curso. También, el 54\% de los encuestados determinaron que su organización del tiempo durante la modalidad virtual fue regular, y cerca del 35\% determinaron su distribución del tiempo como buena. 


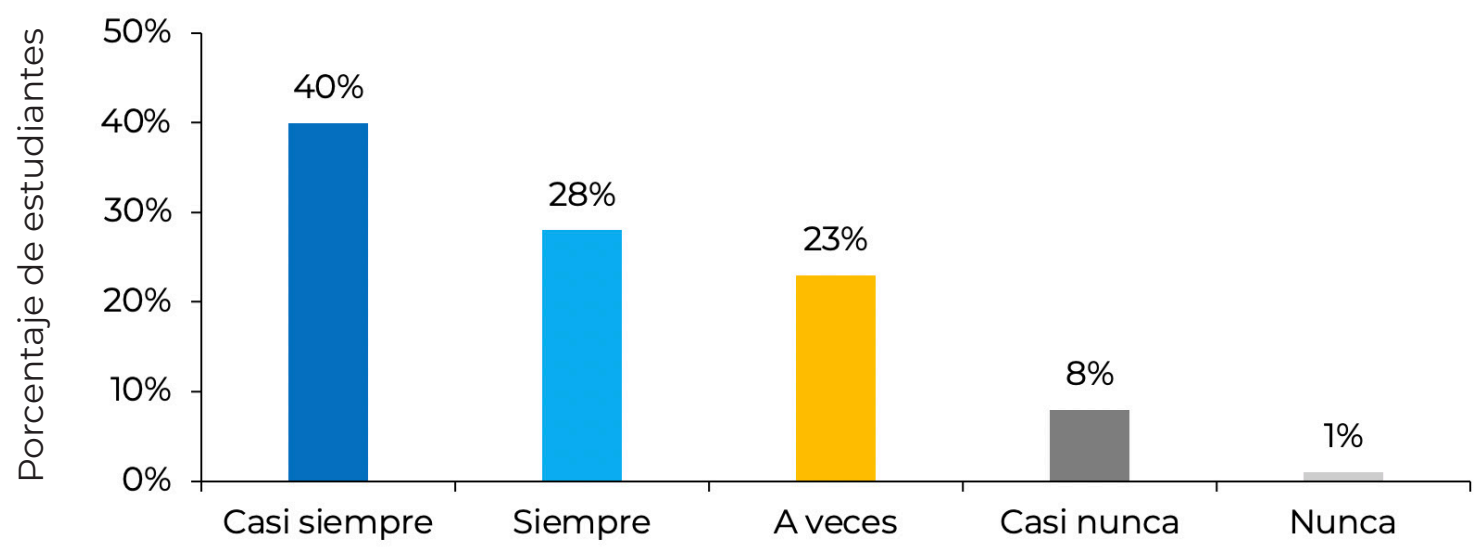

Figura 6. Percepción del nivel de empatía de los profesores ante dificultades de los estudiantes.

Fuente: elaboración propia.

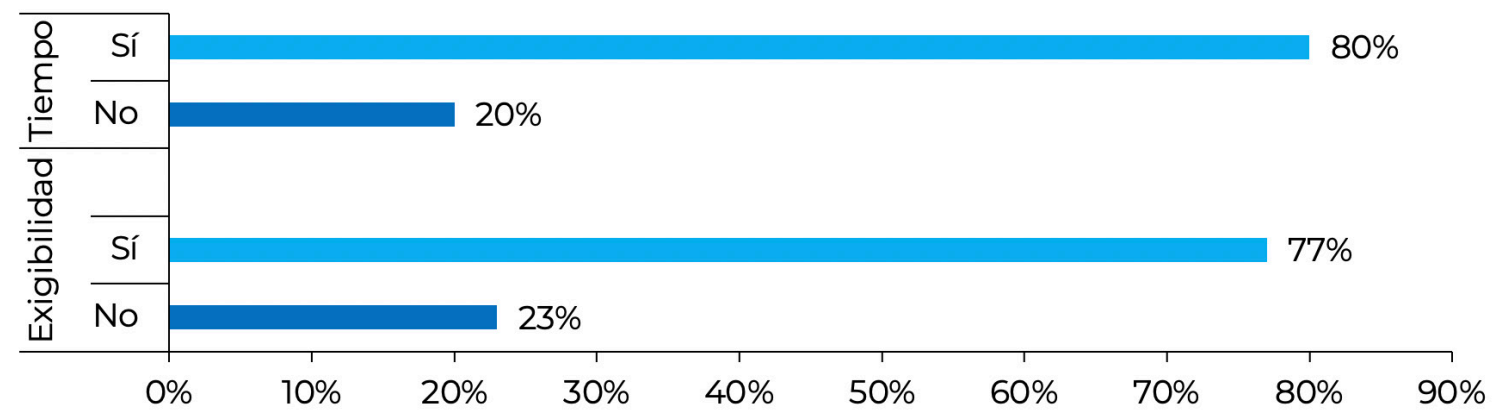

Figura 7. Comparación entre la percepción de los estudiantes respecto a si hubo o no mayor tiempo y exigibilidad durante el I semestre virtual del 2020.

Fuente: elaboración propia.

En una escala del 1 al 10, donde 1 representa la calificación más baja y 10 la calificación más alta, respecto a la influencia en el rendimiento académico, poco más del 50\% de los encuestados determinó un nivel de afectación mayor o igual a 8, el 10 fue la respuesta más seleccionada por los estudiantes. Un 50\% de los estudiantes de la muestra consideran que se sienten intimidados al participar en las clases virtuales más que en las presenciales. En cuanto a los horarios y la forma de impartir las clases, cerca del $70 \%$ de los estudiantes prefieren que haya una combinación entre clases sincrónicas y asincrónicas. 
Cuadro 4. Percepción promedio de los estudiantes de Economía Agrícola y Agronegocios con respecto a tres actividades.

\begin{tabular}{|c|c|c|c|c|c|c|c|c|c|}
\hline \multirow{2}{*}{ Actividad } & \multirow{2}{*}{ Promedio } & \multirow{2}{*}{ Moda } & \multirow{2}{*}{ Max } & \multirow{2}{*}{ Mín } & \multirow{2}{*}{$\mathrm{DE}$} & \multirow{2}{*}{ CV\% } & \multirow{2}{*}{$n$} & \multicolumn{2}{|c|}{ Probabilidad } \\
\hline & & & & & & & & Casol & Caso2 \\
\hline $\begin{array}{l}\text { Adaptación de los } \\
\text { profesores a la } \\
\text { virtualidad }\end{array}$ & 7,56 & 8 & 10 & 3 & 1,76 & 23,34 & 98 & 0,03 & 0,65 \\
\hline $\begin{array}{l}\text { Dificultad del } \\
\text { estudiante para } \\
\text { adaptarse }\end{array}$ & 6,18 & 8 & 10 & 1 & 2,81 & 45,43 & 98 & 0,52 & 0,05 \\
\hline $\begin{array}{l}\text { Nivel de aprendizaje } \\
\text { del estudiante }\end{array}$ & 6,43 & 8 & 9 & 2 & 1,97 & 30,62 & 98 & $0,02^{\ddagger}$ & 0,61 \\
\hline
\end{tabular}

Nota. Caso 1 = Probabilidad según la prueba de Kruskal Wallis para la comparación de los estudiantes entre sedes y recinto. Caso 2 = Probabilidad según la prueba de Wilcoxon para la comparación entre estudiantes que cambiaron de residencia y los que no cambiaron. En este caso no se puede concluir debido a que no se cumple con el supuesto de homogeneidad de las varianzas.

Fuente: elaboración propia.

En la percepción de la adaptación de los docentes, los estudiantes de la Sede del Sur fueron los que dieron una calificación promedio mayor $(8,83)$ y es el único caso en que la diferencia fue estadísticamente significativa $(p=0,03)$. Los estudiantes del recinto de Guápiles dieron una calificación promedio de 7,93, mientras que en la sede Rodrigo Facio fue de 7,27 (Cuadro 4). Referente a la motivación que presentaron los estudiantes respecto a la metodología aplicada, un $65 \%$ de los estudiantes mostraron desmotivación, mientras que un $35 \%$ se vieron motivados. Lo cual conlleva a que un 59\% de los estudiantes están dispuestos a continuar con la modalidad virtual, mientras que un $41 \%$ no lo están.

\section{Discusión}

\section{Aspectos Generales}

En cuanto al año de ingreso de los encuestados a la UCR (Figura 1) se visualizan dos puntos de interés: el primero corresponde a que la mayoría de los estudiantes dentro de la muestra ingresaron en el año 2018. Por otra parte, ocho de los estudiantes ingresaron a la Universidad de Costa Rica en el año 2020. Lo anterior se debe tomar en cuenta si se desea profundizar en los cambios que tuvieron que implementar, considerando que no tenían experiencia previa. Con los resultados expuestos anteriormente se logra 
visualizar que los estudiantes ingresados en el año 2020 a la UCR no han percibido la experiencia universitaria presencial. Según la UNESCO (2020) estas personas no han tenido la oportunidad de desarrollar sus habilidades sociales en ese entorno, lo que les genera una desventaja significativa y puede llegar a ser un problema más grave en cuanto más amplio sea este periodo de confinamiento.

En las figuras 2, 3 y 4 se aprecian los cambios de residencia que los estudiantes experimentaron debido a la pandemia y al cese de clases presenciales. Se realizaron tres mapas que muestran la distribución de los estudiantes en el país, antes de la pandemia, durante el I semestre, 2020 y el II semestre, 2020. La figura 2 muestra que los dos cantones con mayor concentración de estudiantes antes de la pandemia fueron Montes de Oca y Pococí, cantones donde se encuentran localizadas la sede Rodrigo Facio y el recinto de Guápiles respectivamente. Asimismo, se aprecia una concentración no menos significativa de estudiantes en la zona Sur del país, debido a la presencia de la sede del Sur. Por consiguiente, la figura 3 manifiesta los cambios de residencia que ocasionó la pandemia y la modalidad virtual. Se logra apreciar una importante migración del Gran Área Metropolitana (GAM) hacia cantones rurales. Para este caso, provincias como Puntarenas, Guanacaste, Limón y Alajuela, aumentaron la concentración de estudiantes, mientras que San José, Heredia y Cartago disminuyeron. La provincia de San José pasó de albergar aproximadamente el 39\% de los universitarios a un 33\%; mientras que la provincia de Guanacaste pasó de 1\% a 4\%. A su vez, en la figura 4 se manifiesta una mayor distribución de los estudiantes en diferentes zonas del país para el semestre II 2020. Los estudiantes aseguraron que vivir cerca de la casa de enseñanza no era preciso, a causa de la ausencia de las clases presenciales y decidieron regresar a las áreas donde se localizaban su núcleo familiar.

En relación con los cambios de residencia, se puede evidenciar que muchos de los encuestados se trasladaron de su lugar natal para poder cursar su educación superior. Sirva de ejemplo la figura 4 en la que en la provincia de San José pasó de residir un 33 a un 35\% aproximadamente de la totalidad de los encuestados, siendo el principal motivo el movimiento de los estudiantes al cantón de Pérez Zeledón. No obstante, las demás provincias siguieron conservando un escenario similar al presentado en la figura 3. Existen múltiples razones que explican los cambios de residencia de los estudiantes. Por ejemplo, uno de los estudiantes entrevistados explicó que debido a su necesidad de seguir percibiendo la beca por reubicación geográfica debió mantenerse en Montes de Oca para no perder este beneficio. Mientras que una estudiante explicó que en su hogar no lograba concentrarse en sus estudios, esto por motivo de ruido, problemas familiares y personales, por lo que tuvo que trasladarse a otra residencia. 


\section{Aspectos Laborales}

En la parte laboral, Ilama la atención como algunos estudiantes manifiestan estar trabajando en el negocio familiar, lo cual es muy beneficioso considerando el contexto socioeconómico en el que la pandemia ha colocado al sector comercial nacional. Sin embargo, después del primer semestre del 2020, dos estudiantes abandonaron los estudios. Tal como lo menciona Bazán (2020), esto sucede por falta de recursos económicos o por inacceso a las tecnologías requeridas para recibir clases en línea.

\section{Aspectos Cotidianos}

Para evaluar la afectación de los estudiantes en ciertas actividades se utilizó una escala Likert de 10 puntos, en donde 1 representa una baja afectación y 10 que ha habido una alta afectación. En el cuadro 3 se puede observar que en promedio una de las actividades que tuvo mayor afectación en los estudiantes son los pasatiempos. Asimismo, las actividades con mayor coeficiente de variación fueron los horarios de comida y el tiempo en familia, lo cual representa una mayor dispersión en las respuestas. Sin embargo, con las pruebas Kruskal Wallis y Wilcoxon no se encontró una diferencia significativa en la afectación de las actividades, lo que demuestra que las medidas de virtualidad que la universidad aplicó han afectado de manera muy similar a todos los estudiantes, indiferentemente de su sede o recinto.

Asimismo, con respecto a invertir tiempo en otras actividades, los estudiantes de la UCR invirtieron tiempo en actividades físicas principalmente, lo cual coincide con el estudio de Buri et al. (2020) en donde un 21,7\% de los estudiantes de la Universidad Católica de Cuenca Sede Azogues expresó realizar actividad física. Además, en relación con la oportunidad de crear huertas, Alfaro (2020) menciona que esta fue una medida inmediata implementada debido a la pandemia para el autoabastecimiento de alimentos en Costa Rica y hace referencia a que, en las zonas urbanas, las huertas verticales son una práctica que sirve para aprovechar el espacio, además expresa que esta implementación por parte de los estudiantes es una buena práctica para la salud mental.

Con respecto a la afectación en la salud mental y física, existen estudios que coinciden con los resultados encontrados. En el caso de la salud mental, están los estudios de Cao et al. (2020) y el de Ozamiz et al. (2020), en el primer caso se mostró un incremento en problemas de salud mental de estudiantes de Universidades de China, por ejemplo, de la ansiedad debido a la ausencia de comunicación interpersonal. De igual manera en el segundo caso se evidencia que la población entre la edad de los 18 y 25 años presenta una mayor afectación de estos problemas de salud mental, principalmente por la adaptación al nuevo contexto, el estrés ocasionado por la exigencia académica y las evaluaciones de manera virtual. En cuanto a la salud física, en el estudio de Celis 
et al. (2020) se evidencia que el "comportamiento sedente es una reacción inevitable del aislamiento social y las actuales condiciones de confinamiento, sus consecuencias a corto y mediano plazo podrían ser más severas" (Celis et al., 2020, p.885).

\section{Aspectos Académicos}

El hecho de que algunos estudiantes decidieran retirar algún curso puede estar relacionado a muchos factores, entre los cuales podrían estar, según Ozamiz et al. (2020), mayores niveles de estrés, ansiedad y depresión debido al COVID-19. Además, una distribución del tiempo "regular" en la modalidad virtual, en más de la mitad de la muestra (54\%), se debe, según Aristovnik et al. (2020), a que uno de los principales retos que implica la virtualidad, es la autodisciplina. En cuanto a los que determinaron la distribución del tiempo como "buena", esta podría relacionarse con lo especificado por Gonzalez et al. (2020), el cual determina a esta modalidad virtual más beneficiosa para algunos por la nueva metodología utilizada, debido a que posibilita a los estudiantes tener a disposición la materia en cualquier momento del día y estudiarla en el mejor momento posible.

En la Figura 6 se observa un panorama positivo en donde los estudiantes consideran que los profesores fueron empáticos con sus dificultades la mayoría del tiempo. No obstante, en algunos casos los profesores no lo fueron, principalmente con problemas de conexión. Cabe resaltar el hecho de que la UCR realiza grandes esfuerzos para ayudar a la población estudiantil a tener igualdad de oportunidades y que las situaciones socioeconómicas no limiten la calidad de la educación, por ejemplo, con la implementación del sistema de préstamo de tabletas para los estudiantes que no contaban con el equipo electrónico necesario para recibir clases de manera virtual (UCR, 2020). Con respecto a la figura 7 los estudiantes coinciden en que las clases resultan más demandantes en la modalidad virtual que en la presencial. Además, los resultados acerca de la influencia en el rendimiento académico coinciden con los presentados por Aristovnik et al. (2020) "Estudiar desde casa comúnmente requiere una mayor autodisciplina y motivación para seguir lecciones en línea" (p.9). Como se menciona en el artículo, al no estar habituados a la nueva modalidad esta puede generar una sensación de sobrecarga en las obligaciones académicas.

En cuanto a la cantidad de estudiantes de la muestra que mencionaron sentirse más intimidados al participar de manera virtual, se evidencia que uno de los factores que pueden influir es que no se logra el mismo nivel de interacción que ofrece la presencialidad, afectando las relaciones interpersonales y que las personas pueden evadir con mayor facilidad su participación y compromiso. Entre las razones que mencionaron los encuestados respecto a preferir una combinación entre clases sincrónicas y asincrónicas es que no en todos los casos es necesario que el profesor utilice tanto tiempo para impartir las lecciones (De Oliveira-Dias, De Oliveira-Albergarias-Lopes \& 
Correia-Teles, 2020). Por otro lado, hay algunos cursos que se complementan mejor cuando el estudiante realiza el estudio de manera individual y utiliza como complemento ciertos espacios para interactuar con el profesor. Otra razón de gran relevancia para los encuestados es que con las clases asincrónicas tienen la facilidad de que pueden ver los videos en cualquier momento y de esta forma distribuir de mejor manera el tiempo que le dedican al estudio.

Referente al cuadro 4, se les consultó a los estudiantes su percepción con respecto a la adaptación de los profesores de Economía Agrícola a la virtualidad en una escala de 10, siendo 10 la mejor calificación. Los estudiantes consideraron que, en promedio, su nivel de adaptación fue de 7,56. Así mismo, se observa el menor porcentaje del coeficiente de variación, el cual demuestra que hay menor variabilidad en las respuestas, por lo que la percepción de los estudiantes varia en menor grado. Simultáneamente, al realizar la prueba de Kruskal Wallis, se observa que los estudiantes de la sede del sur en promedio percibieron que sus profesores lograron adaptarse de mejor manera a la virtualidad

Por otro lado, el nivel de dificultad que experimentaron los estudiantes para adaptarse a la virtualidad es donde se obtuvieron los resultados con mayor grado de variabilidad. Esto se puede deber al conocimiento previo del manejo de herramientas como las tecnologías de información y comunicaciones (TICS), además de contar con las condiciones óptimas y, principalmente, acceso a internet. De hecho, según la UNESCO (2020), la conectividad de los hogares es uno de los factores determinantes para la adecuada adaptación de los estudiantes a las clases virtuales y en Costa Rica aproximadamente un $80 \%$ de los hogares cuenta con conexión a internet. Teniendo en cuenta lo anterior, y según la moda, los estudiantes percibieron una dificultad de adaptación de 8, en una escala de 10, siendo 10 una excelente adaptación. Este es un nivel de adaptación positivo y deja entrever que los estudiantes, pese a lo inesperado que fue el cierre, lograron adaptarse.

Con respecto al último rubro del cuadro 4, en promedio, el nivel de aprendizaje en las clases virtuales fue de 6,43. Este promedio puede ser explicado debido a que las mallas curriculares y la metodología de los cursos no fue diseñada para la modalidad virtual. Además, tal como lo menciona la UNESCO (2020) los estudiantes requieren de un alto grado de disciplina y compromiso para poder sobrellevar de manera exitosa la modalidad virtual. Por otro lado, los encuestados expusieron sus razones por las cuales la metodología les motivaba o no. Las desmotivaciones por parte de la gran mayoría de los encuestados (65\% de los estudiantes) fueron principalmente la extensión de los videos, la poca capacitación de los profesores en modalidad virtual, la falta de comunicación con los estudiantes y la monotonía de los docentes. Mientras que, las motivaciones de la minoría de los encuestados (35\% de los estudiantes) fueron la generación de una mayor capacidad analítica en las dinámicas de los cursos y no tanto sistemática como lo era en las clases presenciales, el uso de herramientas digitales aumentó la capaci- 
dad creativa, un desarrollo más interactivo y una mejor comprensión de la materia de los cursos en ciertas ocasiones.

Así mismo, dentro de los retos percibidos por los estudiantes en la modalidad virtual en comparación a la modalidad presencial se encontraban las distracciones, la dificultad de comprensión, organización del tiempo y problemas de conectividad. Un 59\% de la muestra de estudiantes consideran que estarían dispuestos a continuar con las clases virtuales. Algunos de sus argumentos son que les gustaría una combinación entre las clases virtuales y presenciales, ejemplo: "Las clases podrían ser alternadas, además hay ciertas clases que podría ser más provechosas de forma virtual, en relación con inversión en pases, alimentación y tiempo". Mientras que el otro 41\% no estarían dispuestos a continuar con las clases en modalidad virtual. Otro de los argumentos utilizados es"No vale la pena sacrificar la salud física o mental por mantener clases virtuales. Claro, dependería de los profesores y del curso en sí.". Sin embargo, no descartan la posibilidad de la combinación de ambas modalidades. Los estudiantes calificaron del 1 al 10 las medidas y adaptaciones a la virtualidad por parte de la UCR, donde 1 es la calificación más baja y 10 la más alta, 7 fue la evaluación más escogida por los estudiantes para valorar la respuesta de la UCR ante la emergencia nacional.

\section{Conclusiones}

La pandemia ha hecho evidentes los retos que presenta la educación, se denota la necesidad de adaptarse al nuevo contexto en donde el uso de las tecnologías es una herramienta cada vez más imprescindible. Además, se evidencia que los cambios que han tenido que afrontar los estudiantes no se limitan al área académica, si no que trascienden al entorno personal de estos, como lo son su salud mental y física. Ante esto, la educación superior debe de priorizar la inclusión de los estudiantes a la modalidad virtual considerando aspectos como residencia, situación económica y laboral. Para esto es primordial brindar un seguimiento tanto a los estudiantes como a los docentes, con el fin de conocer la perspectiva y cambios que han tenido que implementar debido a la virtualidad y dar una respuesta asertiva ante posibles dificultades en el proceso.

Es de suma importancia relacionar los resultados obtenidos con la formación de los futuros profesionales en Economía Agrícola y Agronegocios, reconocer que la educación virtual tiene falencias y que estas pueden afectar sus futuras decisiones. Se debe procurar la calidad de la enseñanza, asegurándose que los estudiantes verdaderamente estén adquiriendo los conocimientos necesarios, aunque esto signifique reevaluar las metodologías de evaluación. El presente trabajo es una muestra de que incluso en la virtualidad los estudiantes pueden aplicar el conocimiento adquirido y aportar a la sociedad. 
Además, se recomienda a las instancias que tengan la capacidad, facilitar herramientas, asesoría y capacitaciones a los docentes, para que participen activamente junto con las universidades con el fin de mejorar el modelo pedagógico impartido en esta modalidad y adaptar el perfil del personal docente a la nueva normalidad. Por último, se resalta la importancia documental de este estudio, que recopila información de fuente primaria de un evento trascendental para la historia, enfocado hacia la experiencia de los estudiantes de Economía Agrícola y Agronegocios, exponiendo su voz.

\section{Literatura citada}

Alfaro, D. (2020, mayo 13). Huertas urbanas: autoabastecimiento durante la pandemia por el COVID-19. Universidad de Costa Rica. https://www.ucr. ac.cr/noticias/2020/05/13/huertas-urbanas-autoabastecimiento-durante-lapandemia-por-el-covid-19.html

Aristovnik, A; Kerži ` , D; Ravšelj, D; Tomaževi `c, N \& Umek, L. (2020). Impacts of the COVID-19 Pandemic on Life of Higher Education Students: A Global Perspective. Sustainability, 12(20), 8438. https://doi.org/10.3390/ su12208438

Bazán, H. (2020, mayo 14). La educación universitaria y su respuesta a la crisis del covid-19: Continuidad, adaptación e innovación. UPAGU. https://upagu.edu. pe/es/la-educacion-universitaria-y-su-respuesta-a-la-crisis-del-covid-19continuidad-adaptacion-e-innovacion/

Buri, F. N. A., León, D. A. H., Mediavilla, C. M. Á., \& Navarro, W. H. B. (2020). La actividad física en estudiantes universitarios antes y durante la pandemia COVID-19. Polo del Conocimiento: Revista científico-profesional, 5(11), 163176.

Cao, W., Fang, Z., Hou, G., Han, M., Xu, X., Dong, J., \& Zheng, J. (2020). The psychological impact of the COVID-19 epidemic on college students in China. Psychiatry Research, 287, 112934. https://doi.org/10.1016/j. psychres.2020.112934

Celis-Morales, C., Salas-Bravo, C., Yáñez, A., \& Castillo, M. (2020). Inactividad física y sedentarismo. La otra cara de los efectos secundarios de la Pandemia de COVID-19. Revista médica de Chile, 148(6), 885-886. 
CEPAL. (2020). América Latina y el Caribe ante la pandemia del COVID-19. https:// repositorio.cepal.org/bitstream/handle/11362/45337/4/S2000264_es.pdf

De Oliveira-Dias, M., De Oliveira-Albergarias-Lopes, R., \& Correia-Teles, A. (2020). Will Virtual Replace Classroom Teaching? Lessons from Virtual Classes via Zoom in the Times of COVID-19. Journal of Advances in Education and Philosophy, 4(05), 208-213. www.doi.org/10.36348/jaep.2020.v04i05.004

Gaiser, E. (2020). Atravesando la crisis con un espíritu de innovación. Fundación Konrad Adenauer Costa Rica. https://www.kas.de/documents/273967/8233910/ Atravesando+la+crisis+con+un+esp\%C3\%ADritu+de+innovaci\%C3\%B3n. pdf/84leb70b-a674-7d2b-2dff-a65cac440e2a?version=1.0 $\& t=1588689139037$

Gonzalez, T., De La Rubia, M. A., Hincz, K. P., Comas-Lopez, M., Subirats, L., Fort, S., \& Sacha, G. M. (2020). Influence of COVID-19 confinement on students' performance in higher education. PloS one, 15(10), e0239490.

Huanca-Arohuanca, J., Supo-Condori, F., Leon, R. S., \& Quispe, L. S. (2020). El problema social de la educación virtual universitaria en tiempos de pandemia, Perú. Innovaciones Educativas, 22, 115-128.

Johnson, M., Saletti, L., \& Tumas, N. (2020). Emociones, preocupaciones y reflexiones frente a la pandemia del COVID-19 en Argentina. Ciência \& Saúde Coletiva, 25(1.1) 2447-2456. https://scielosp.org/pdf/csc/2020. v25suppl1/2447-2456/es

Kikut, L. (2020). Análisis de resultados de la evaluación de la virtualización de cursos en la UCR ante la pandemia por COVID-19: Perspectiva estudiantil. Universidad de Costa Rica.

Kruskal, W., \& Wallis, W. (1952). Use of Ranks in One-Criterion Variance Analysis. Journal of the American Statistical Association, 47(260), 583-621. www.doi. org/10.2307/2280779

Levene, H. (1960). In Contributions to Probability and Statistics: Essays in Honor of Harold Hotelling, I. Olkin et al. eds., Stanford University Press, 278-292.

Montalvo, N., \& Montiel, A. (2020). IMPACTO DEL COVID-19 EN EL ESTRÉS DE UNIVERSITARIOS. http://itchetumal.edu.mx/avacient/index.php/revista/article/ download/132/102. 
Mora, M. (2015). Formulas y tablas estadísticas. Universidad de Costa Rica.

Ozamiz-Etxebarria, N., Dosil-Santamaria, M., Picaza-Gorrochategui, M. \& IdoiagaMondragon, N. (2020). Niveles de estrés, ansiedad y depresión en la primera fase del brote del COVID-19 en una muestra recogida en el norte de España. Cadernos de Saúde Pública, 36(4). https://www.scielo.br/scielo.php?pid=S0102311X2020000405013\&script=sci_abstract\&tlng=es

Peña, R., Waldman, F., Soneyra, N., Tejada, G., Carrere-Cadirant, G., Passaglia, J., \& Contrera, M. (2012). Implementación de los entornos virtuales de aprendizaje en cursos de capacitación docente. Revista Iberoamericana de educación, 60(2012), 117-128. https://rieoei.org/historico/documentos/rie60a07.pdf

Pérez-López, E., Atochero, A. V. \& Rivero, S. C. (2021). Educación a distancia en tiempos de COVID-19: Análisis desde la perspectiva de los estudiantes universitarios. Revista Iberoamericana de Educación a Distancia, 24(1), 331350.

UNESCO (2020). COVID-19 y educación superior: De los efectos inmediatos al día después. ONU.

Universidad de Costa Rica. (2020, septiembre 23). La UCR robustece mecanismo de préstamo de tabletas a estudiantes para el II ciclo lectivo 2020. https://www. ucr.ac.cr/noticias/2020/09/23/la-ucr-robustece-mecanismo-de-prestamo-detabletas-a-estudiantes-para-el-ii-ciclo-lectivo-2020.html

Wilcoxon, F. (1945). Individual Comparisons by Ranking Methods. Biometrics Bulletin, 1(6), 80-83. www.doi.org/10.2307/3001968 